\title{
How should we move for health? The case for the 24-hour movement paradigm
}

\author{
Mark S. Tremblay PhD, Robert Ross PhD
}

\author{
— Cite as: CMAJ 2020 December 7;192:E17298-9. doi: 10.1503/cmaj.202345
}

$\mathbf{R}$ ecognition of the health effects of insufficiently active lifestyles around the globe has provoked the development and release of public health guidelines to support strategies to increase physical activity. In November 2020, the World Health Organization (WHO) released new guidelines on physical activity and sedentary behaviour, ${ }^{1}$ updating their earlier guidelines from 2010 and closely matching the physical activity guideline released in 2018 by the US Department of Health and Human Services. ${ }^{2}$ In October 2020, the Canadian 24-Hour Movement Guidelines for Adults aged 18-64 years and Adults aged 65 years or older: An Integration of Physical Activity, Sedentary Behaviour, and Sleep ${ }^{3}$ were released. Although the WHO and the Canadian guidelines are similar in many respects, there are notable and important differences, which warrant explanation for those who may find the differences confusing. We consider the differences between the 2 recently published guidelines, explain the alternate approaches these guideline groups have taken and discuss how differing guidelines can coexist.

The differences between the recent WHO and Canadian guidelines reflect a global divide in the approach to movement behaviour guideline development. ${ }^{1-4}$ Briefly, the recent Canadian guidelines consider movement over a 24-hour period, whereas the new WHO guidelines focus on moderate- to vigorous-intensity physical activity (MVPA) and sedentary behaviour. Furthermore, the Canadian guidelines make specific recommendations for light physical activity, total daily sedentary time and total daily recreational screen time, and provide guidance in relation to integrated movement behaviour tradeoffs (e.g., emphasizing that reallocating more time into MVPA from any of the other movement behaviours is associated with greater health benefits); in contrast, the WHO guidelines provide specific recommendations for MVPA only.

In the past few years, different approaches have emerged for the development of movement behaviour guidelines, and 2 dominant differences have become evident. First, increasing awareness of and focus on the importance of considering movement behaviours throughout the day (i.e., physical activities of all intensities, sedentary behaviours and sleep) and their integrated effect on health, has contrasted with a focus on only activity of certain intensity. ${ }^{3-5}$ Moreover, guideline producers have considered and continue to debate the relative merits of making specific

\section{KEY POINTS}

- The World Health Organization (WHO), Canada and other countries have recently released public health guidelines related to healthy movement behaviours (e.g., physical activity and sedentary behaviours).

- Despite using similar robust approaches to guideline development, notable differences are evident between the recent $\mathrm{WHO}$ and Canadian guidelines.

- In general, the WHO guideline group focused on moderateto vigorous-intensity physical activity and sedentary behaviour, whereas the Canadian group took an integrated 24-hour movement behaviour approach, including physical activity of all intensities, sedentary behaviours, screen time and sleep behaviours.

- The 24-hour movement guideline approach used by the Canadian guideline group will likely lead to more nuanced benchmarks for surveillance, and should result in additional research to inform future guideline revisions.

(e.g., "limiting sedentary time to 8 hours or less" per day3) versus more general ("limit the amount of time being sedentary"1 or "sit less throughout the day"2) recommendations, with active debate regarding the approach to be taken. ${ }^{6,7}$ The new WHO guidelines and the Canadian 24-hour guidelines for age-matched groups exemplify these different approaches, as summarized in Appendix 1, available at www.cmaj.ca/lookup/doi/10.1503/ cmaj.202345/tab-related-content. However, they also share many similarities, especially relating to guidance on MVPA.

The 2 authoritative guidelines have drawn on the same underlying evidence base. Their different recommendations reflect differences in the guideline development panels' consideration of the evidence. Both guideline development processes adhered to the Grading of Recommendations Assessment, Development, and Evaluation (GRADE) framework ${ }^{8}$ and their detailed findings, study quality assessments and evidence-to-decision explanations are provided in their background materials. ${ }^{1,3}$ Both sets of guideline recommendations and their specific wording are explained and justified, with the differences resulting from accepting or rejecting certain available research evidence. Although the underlying literature remains the same, the specific guideline recommendations differ mainly because of variations 
in the degree to which lower-quality evidence was considered and incorporated.

The panel responsible for drafting the Canadian guidelines used a 24-hour movement paradigm and thus considered how all types of activity, sedentary behaviours and sleep are related to health, even though evidence related to some of these dimensions is of lesser quality. Although it is desirable to use only high-quality evidence to inform guidelines, recommendations in the absence of high-quality evidence are preferable to no recommendations at all, provided that potential benefits outweigh potential harms. ${ }^{9}$ Patients and providers prefer guidance based on the best available evidence, even if the evidence is of low quality. Both the Canadian and recent WHO guidelines recognize the unequivocal importance of MVPA. However, the Canadian guidelines acknowledge, speak to and provide guidance for light physical activity, as well as daily sedentary behaviour volume and recreational screen time. The Canadian guideline group's 24-hour movement approach recognizes the codependence and interactions among movement behaviours of different intensities, and that movement throughout the whole day matters for health. ${ }^{5}$ For example, the guidelines provide the practitioner with options to suggest to patients when advising them to move more (e.g., aerobic activities, strengthening activities and light activities including standing), reduce sedentary time (e.g., limit recreational screen time and break up long periods of sitting) and sleep well (e.g., regular bedtime routines and consistent bed and wake-up times). ${ }^{3}$ This approach has global momentum, ${ }^{4}$ with the $\mathrm{WHO}$ adopting it for their recently released guidelines for the early years. ${ }^{10}$

These differences in movement recommendations may lead to different public health and population surveillance approaches among jurisdictions. Setting more nuanced benchmarks for surveillance will likely result in additional research to inform future guideline revisions, while offering guidance for developing and evaluating interventions that promote a balanced lifestyle, and accelerating implementation research and future scale-up of strategies to promote an active lifestyle. Although the current evidence informing movement guidelines is and may always be imperfect, the creators of the Canadian guidelines consider that interpretation of that evidence must lead to advice that has the best chance of preserving and promoting people's health by providing practitioners with more options to explore with patients in a patient-centred way, in an effort to improve health through modification of movement behaviour.

\section{References}

1. Bull FC, Al-Ansari SS, Biddle S, et al. World Health Organization 2020 guidelines on physical activity and sedentary behaviour. Br J Sports Med. 2020 Nov. 26 [Epub ahead of print]. doi:10.1136/bjsports-2020-102955.

2. 2018 Physical Activity Guidelines Advisory Committee. 2018 Physical Activity Guidelines Advisory Committee scientific report. Washington (DC): US Department of Health and Human Services; 2018.

3. Ross R, Chaput J-P, Giangregorio LM, et al. Canadian 24-hour movement guidelines for adults aged 18-64 years and adults aged 65 years or older: an integration of physical activity, sedentary behaviour, and sleep. Appl Physiol Nutr Metab Oct;45:S57-S102.

4. Tremblay MS. Introducing 24-hour movement guidelines for the early years: a new paradigm gaining momentum. J Phys Act Health 2020;17:92-5.

5. Rollo S, Antsygina O, Tremblay MS. The whole day matters: understanding 24-hour movement guideline adherence and relationships with health indicators across the lifespan. J Sport Heal Sci 2020 July 21 [Epub ahead of print]. doi: 10.1016/j.jshs.2020.07.004.

6. Stamatakis E, Ekelund U, Ding D, et al. Is the time right for quantitative public health guidelines on sitting? A narrative review of sedentary behaviour research paradigms and findings. Br J Sports Med 2019;53:377-82.

7. Chaput JP, Olds T, Tremblay MS. Public health guidelines on sedentary behaviour are important and needed: a provisional benchmark is better than no benchmark at all. Br J Sports Med 2020;54:308-9.

8. Guyatt GH, Oxman AD, Vist GE, et al. GRADE: What is "quality of evidence" and why is it important to clinicians? BMJ 2008;336:995-8.

9. Neumann I, Schünemann HJ. Guideline groups should make recommendations even if the evidence is considered insufficient. CMAJ 2020;192:E23-4.

10. Willumsen J, Bull F. Development of WHO guidelines on physical activity, sedentary behavior and sleep for children less than 5 years of age. J Phys Act Health 2020;17:96-100.

Competing interests: Robert Ross received funding from Queen's University and the Public Health Agency of Canada during the conduct of the study. He also was the chairperson of the Guideline Development Panel for the Canadian 24-hour movement guidelines for adults. Mark Tremblay was a volunteer member of the Guideline Development Panel for the Canadian 24-hour movement guidelines for adults. No other competing interests were declared.

This article has been peer reviewed.

Affiliations: CHEO Research Institute, and Department of Pediatrics (Tremblay), Faculty of Medicine, University of Ottawa, Ottawa, Ont;; School of Kinesiology and Health Studies, Faculty of Arts and Sciences, and School of Medicine, Division of Endocrinology and Metabolism (Ross), Queen's University, Kingston, Ont.

Contributors: Mark Tremblay conceived of and drafted the manuscript. Robert Ross critically reviewed and edited the commentary for content and accuracy. Both authors gave final approval of the version to be published and agreed to be accountable for all aspects of the work.

Correspondence to: Mark Tremblay,mtremblay@cheo.on.ca 